\title{
Caffeine stimulates the proliferation of human lung adenocarcinoma cells and small airway epithelial cells via activation of PKA, CREB and ERK1/2
}

\author{
HUSSEIN A.N. AL-WADEI ${ }^{1}$, TAKASHI TAKAHASHI ${ }^{2}$ and HILDEGARD M. SCHULLER ${ }^{1}$ \\ ${ }^{1}$ Experimental Oncology Laboratory, Department of Pathobiology, College of Veterinary Medicine, University of Tennessee, \\ Knoxville, TN, USA; ${ }^{2}$ Division of Molecular Carcinogenesis, Center for Neurological Diseases and Cancer, \\ Nagoya University Graduate School of Medicine, Nagoya, Japan
}

Received September 1,2005; Accepted October 17, 2005

\begin{abstract}
The incidence of pulmonary adenocarcinoma (PAC) has increased dramatically over the last three decades. Recent studies have shown that human PAC cells with phenotypic features of bronchiolar Clara cells and experimentally induced PAC of Clara cell origin are under $B$-adrenergic growth control. The phosphodiesterase inhibitor, theophylline, which is contained in tea, asthma/allergy medications and numerous dietary supplements selectively stimulated the growth of this cancer type in vivo and in vitro. The current study has tested the hypothesis that another environmentally prominent phosphodiesterase inhibitor, caffeine, has similar effects. Using a cell line derived from a human PAC with Clara cell features (PACC) and immortalized human small airway epithelial cells (SAECs), our data show that caffeine activated protein kinase A (PKA), the mitogen-activated kinases ERK1/2, the nuclear transcription factor cyclic AMP response element binding protein (CREB) and stimulated cell proliferation in these cell lines. These findings suggest that exposure to caffeine may contribute to the prevalence of PAC observed today.
\end{abstract}

\section{Introduction}

Lung cancer is the leading cause of cancer death in both, men and women, and cigarette smoking is a dominant risk factor for this disease (1). Pulmonary adenocarcinoma (PAC) accounted for less than $10 \%$ of all lung cancer cases 30 years ago but is now the leading type of lung cancer in smokers and nonsmokers (2-4). Human PAC may be derived from bronchiolar Clara cells or from alveolar type II cells (5). Immunohistochemistry is primarily used to identify cell lineage of this

Correspondence to: Dr H.M. Schuller, Department of Pathobiology, College of Veterinary Medicine, University of Tennessee, 2407 River Drive, Knoxville, TN 37996, USA

E-mail: hmsch@utk.edu

Key words: caffeine human lung adenocarcinoma, PKA/CREB, growth stimulation cancer type by using antibodies to the Clara cell-specific CC10 protein and the alveolar type II cell-specific surfactant. Using this technique, PAC of Clara cell lineage (PACC) has been reported to account for about $50 \%$ of PAC cases (6). By contrast, electron microscopic investigations have identified $90 \%$ of PAC as being derived from Clara cells (7). Recent reports have shown that exposure to cigarette smoke or to the tobacco-specific carcinogenic nitrosamine 4-(methylnitrosamino)-1-(3-pyridyl)-1-butanone (NNK) down-regulate the expression of the Clara cell-specific $\mathrm{CC} 10$ protein in human and animal lungs (8). These findings suggest that identification of PAC cell lineage by immunohistochemistry may result in significant numbers of false negative immunoreactions to the CC10 antibody because of down-regulated CC10 in smokers. Accordingly, a higher proportion of PACs are likely of Clara cell lineage than is generally accepted in the literature.

Studies in our laboratory have shown that the in vitro growth of human lung cancer cell lines derived from PACC are regulated by $\beta$-adrenergic receptors and that the tobaccospecific carcinogenic nitrosamine 4-(methylnitrosamino)-(3pyridyl)-1-butanone (NNK) acts as an agonist for this receptor family (9). NNK as well as a stimulator of the classic downstream effector of $\beta$-adrenergic receptors, cAMP, significantly stimulated DNA synthesis of these cells in vitro $(9,10)$. Studies in a hamster model of NNK-induced PACC have shown that $\beta$-adrenergic agonists and the phosphodiesterase inhibitor, theophylline, which causes intracellular accumulation of cAMP, each significantly promoted the development of this cancer type $(11,12)$. Similarly, hamsters given green tea as the single source of drinking fluid in addition to multiple injections with NNK developed significantly more PACC than animals given NNK alone (12). Analysis by HPLC confirmed the presence of significant amounts of theophylline and caffeine in the green tea used in the hamster study. Subsequently, in vitro experiments showed that theophylline stimulated the proliferation of human PACC cells and their normal cells of origin, small airway epithelial cells (SAECs) via cAMPinitiated activation of protein kinase A (PKA), mitogenactivated protein kinases ERK1/2 and cAMP response element binding protein (CREB) (13). Both, theophylline and caffeine, are methylxanthines that increase intracellular cAMP levels by inhibiting the enzyme phosphodiesterase (14). The current 
experiments have therefore tested the hypothesis that caffeine has similar stimulating effects on the growth of human PACC cells and SAECs as theophylline.

\section{Materials and methods}

Cell lines and tissue culture. The human PAC cell line with characteristics of Clara cells, NCI-H322 (Center for Applied Microbiology and Research; ECACC, Salisbury, Wiltshire, UK) was maintained in RPMI-1640 medium (American Type Culture Collection, Manassas, VA) supplemented with 10\% FBS, $10 \mathrm{mM}$ HEPES, $1 \mathrm{mM}$ sodium pyruvate, $2 \mathrm{mM}$ L-glutamine, $4500 \mathrm{mg} / 1$ glucose, and $1500 \mathrm{mg} / \mathrm{l}$ sodium bicarbonate. The simian virus 40 (SV40)-immortalized human peripheral airway cell line HPL1D is referred to in this publication as small airway epithelial cells SAEC. These cells were maintained in F-12 Nutrient mixture (HAM; Gibco, Carlsbad, CA) medium supplemented with $1 \%$ FBS, $15 \mathrm{mM}$ HEPES pH 7.3, $5 \mu \mathrm{g} / \mathrm{ml}$ insulin, $5 \mu \mathrm{g} / \mathrm{ml}$ transferrin, $10^{-7} \mathrm{M}$ hydrocortisone, $2 \times 10^{-10} \mathrm{M}$ triiodothyronine (Cambrex, Walkersville, MD). Both cell lines were maintained in antibiotic-free medium. Assays with NCI-H322 cells were conducted in low-serum $(0.1 \%)$ RPMI medium while assays with SAECs were conducted in their basal media with $0.05 \%$ FBS without other supplements.

PKA activation assay. Following incubation of cells with caffeine $\left(10 \mathrm{pM}\right.$ in $\mathrm{PBS}$ at $\left.37^{\circ} \mathrm{C}\right)$ for $5-60 \mathrm{~min}$ as specified in the figure legends, PKA activity was assayed in cell lysates using a PepTag assay for non-radioactive detection of activated PKA (Promega Corporation, Madison, WI, USA), following the instruction of the manufacturer. This assay utilizes fluorescent substrate for PKA that changes the peptide's net charge upon phosphorylation of PKA, thus allowing the phosphorylated peptide to migrate to the positive electrode $(+)$, while the non-phosphorylated peptide migrates to the negative electrode (-). Briefly, reactions containing a brightly colored fluorescent peptag A1 peptide $(0.4 \mu \mathrm{g} / \mathrm{ml})$, peptide protection PepTag PKA reaction and PKA activator solutions were incubated in ice for few min before 1-min incubation at $30^{\circ} \mathrm{C}$. After adding samples, cAMP-dependent protein kinase reactions were incubated at room temperature for $30 \mathrm{~min}$, boiled at $95^{\circ} \mathrm{C}$ for $10 \mathrm{~min}$, and loaded onto $0.8 \%$ agarose gel in $50 \mathrm{mM}$ Tris- $\mathrm{HCl}(\mathrm{pH} 8.0)$. At this point the qualitative assay is complete, and the protein kinase A activity in samples is determined by examining the gel under UV lights. Densitometric analysis of the bands was conducted after inversion of the images, using NIH Scion software for image quantitation.

Data are expressed as mean values and standard errors of five densitometric readings per band after background subtraction. Each experiment was repeated once with similar results. Statistical analysis of data was by one-way ANOVA, Tukey-Kramer multiple comparisons test and two-tailed unpaired t-test.

Assessment of total proteins and phosphorylated proteins by Western blotting. To assess the effects of caffeine on the expression and phosphorylation of the mitogen-activated protein kinases ERK1/2 or the cAMP response element binding protein CREB, 500,000 NCI-H322 or SAEC cells were seeded into culture vessels $\left(100 \mathrm{~cm}^{2}\right)$ containing their respective growth media. When the cells had reached $60-65 \%$ confluence, they were rinsed one time with $1 \mathrm{X}$ PBS and serum-starved for $24 \mathrm{~h}$. Following removal of the media and replacement with fresh low-serum media, caffeine (dissolved in PBS) was added to the culture vessels (final concentration $10 \mathrm{pM}$ ) and cells were incubated from 5 to $60 \mathrm{~min}$ as detailed in the figure legends. Cells exposed to the caffeine vehicle (PBS) served as controls. The cultured cells then were washed once with cold PBS, lysed in $20 \mathrm{mM}$ Tris-base, $200 \mathrm{mM} \mathrm{NaCl}, 1 \mathrm{M}$ sodium fluoride, 0.5 M EDTA, $100 \mathrm{mM} \mathrm{Na}_{3} \mathrm{VO}_{4}, 100 \mathrm{mM} \mathrm{PMSF}, 1 \mu \mathrm{l}$ pepstatin, $1 \mu 1$ leupeptin, $1 \mu 1$ aprotinin, and $0.25 \%$ NP-40. Then, protein samples were denatured by boiling at $95^{\circ} \mathrm{C}$ for 5 min, separated on $10 \%$ SDS-PAGE, and transferred to nitrocellulose. Membranes were blocked with 5\% non-fat dry milk, probed with rabbit polyclonal CREB and phospho-CREB antibodies, respectively, and developed by chemiluminescence with ECL reagents membranes were blocked in $10 \mathrm{ml}$ of $5 \%$ non-fat dry milk (Kroger) in $1 \mathrm{X}$ TBST for $1 \mathrm{~h}$. Membranes were then incubated overnight at $4^{\circ} \mathrm{C}$ with primary antibodies at a 1:1000 dilution (rabbit polyclonal for total ERK1/2, rabbit polyclonal for Thr202/Tyr204 phosphorylated Erk1/2; rabbit polyclonal for total CREB, mouse monoclonal for SER33 phosphorylated CREB) (Cell Signaling Technology, Beverly, MA). Densitometric analysis of the bands was conducted, using NIH Scion software for image quantitation.

Data are expressed as mean values and standard errors of five densitometric readings per band. Each experiment was repeated twice and yielded similar data. Statistical analysis of data was by one-way ANOVA, Tukey-Kramer multiple comparisons test and two-tailed unpaired t-test.

Assessment of cell numbers by MTT assay. The effects of caffeine on cell proliferation were assessed by the colorimetric 3-(4,5-dimethylthiazol-2-yl)-2,5-diphenyl-tetrazolium bromide (MTT) assay (Sigma). Briefly, the MTT test is based on the NADH-dependent enzymatic reduction of the tetrazolium salt MTT [3-(4,5-dimethylthiazol-2-yl)-2,5-diphenyl-tetrazoliumbromide] in metabolically active cells but not in dead cells. Cells were seeded into 6-well tissue culture plates (Falcon, Franklin Lakes, NJ, USA) at a density of 50,000 cells per well. The cells were left to grow in complete media at $37^{\circ} \mathrm{C}$ with $5 \% \mathrm{CO}_{2}$ for $5 \mathrm{~h}$ to attach. The cells were then switched to fresh low serum $(0.1 \%$ FBS, NCI-H322) or basal media with $0.05 \%$ FBS without other additives (SAECs) and caffeine dissolved in PBS was added to yield the final concentrations specified in the figure legends and incubated for $72 \mathrm{~h}$. Cells treated with PBS alone served as controls. Three hours before the end of the 72-h incubation period, $100 \mu \mathrm{l}$ of 3 (4,5-dimethylthiazol-2-yl)-2,5-diphenyl-tetrazolium bromide $(0.5 \mathrm{mg} / \mathrm{ml})$ was dissolved in complete medium together with phenol-free medium (Gibco) and added to the cells to allow metabolic conversion of the MTT substrate to blue formazan. The media was replaced with isopropanol, and optical density at $570 \mathrm{~nm}$ was determined using an ELISA reader.

Data are expressed as mean values and standard errors of four samples per treatment group. Each experiment was repeated twice and yielded similar data. Statistical analysis of data was by one-way ANOVA, Tukey-Kramer multiple comparisons test and two-tailed unpaired t-test. 
A
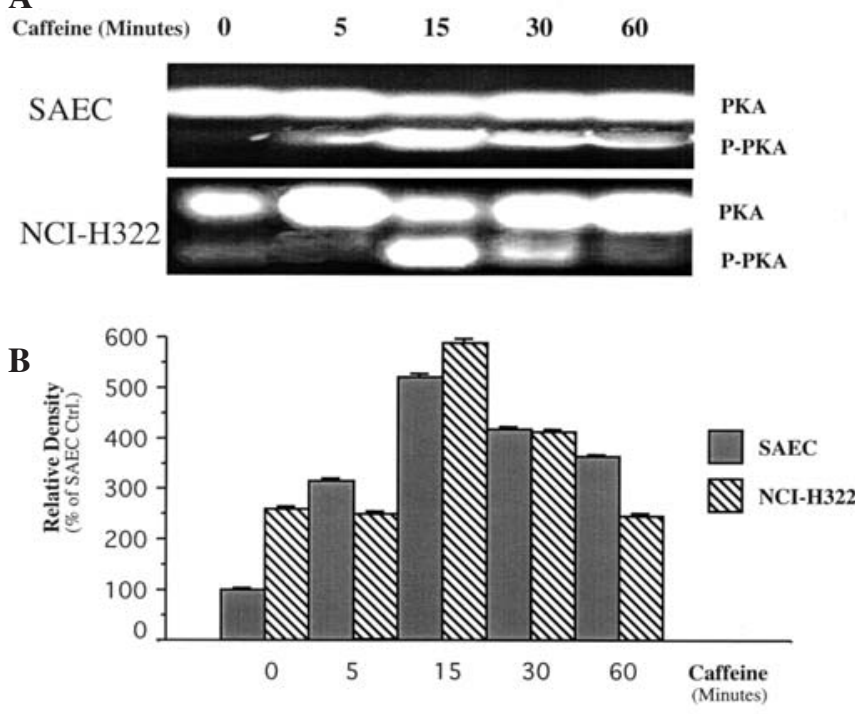

Figure 1. A, Agarose gel showing the effects of caffeine (10 pM) incubated for 5-60 min on phosphorylation of SAEC and NCI-H322. PKA was assayed using a non-radioactive PepTag assay. B, Bar graph demonstrating normalized (controls of SAECs are set as $100 \%$ ) mean values and standard deviations of five densitometric readings per band. The experiment was repeated twice with similar results. Statistical analysis was by one-way ANOVA, Tukey-Kramer multiple comparison test and two-tailed unpaired t-test. The increases in PKA phosphorylation were significant $(\mathrm{P}<0.001)$ at all time intervals tested in SAECs and at the 15- and 30-min time intervals in NCI-H322.

\section{Results}

PKA is the classic downstream effector activated by cAMP in mammalian cells (15). In light of the documented ability of caffeine to inhibit phosphodiesterases that mediate the cellular breakdown of cAMP (14), we first tested the hypothesis that caffeine activates PKA in PACC and SAECs. As shown in Fig. 1, SAECs exposed to the solvent of caffeine, PBS, demonstrated no detectable PKA activity whereas PBSexposed NCI-H322 cells had significant levels of basal PKA activity. Both cell lines responded to caffeine $(10 \mathrm{pM})$ with significant $(\mathrm{P}<0.01)$ increases in PKA activity, with peak levels (5.2-fold increase for SAECs, 2.3-fold increase for NCI-H322 cells) observed after 15 min of exposure (Fig. 1).

Activation of PKA generally leads to phosphorylation of the transcription factor CREB in mammalian cells (15). On the other hand, cross-talk between the cAMP/PKA/CREB pathway and the mitogen-activated kinase (MAPK) pathway, including the extracellular signal regulated kinases (ERK1/2) has been reported in several cell types $(16,17)$. We therefore tested the hypothesis that caffeine activates CREB and ERK1/2 in PACC and SAECs. Western blot analysis showed that caffeine $(10 \mathrm{pM})$ significantly $(\mathrm{P}<0.01)$ increased the levels of phosphorylated ERK1/2 of SAECs in a time-dependent manner (Fig. 2), with peak levels (2.4-fold) observed after $15 \mathrm{~min}$ of exposure. Similarly, caffeine significantly $(\mathrm{P}<0.01)$ induced the expression levels of phosphorylated CREB over time in these cells, with the highest levels (2.3-fold) observed after 5 and $15 \mathrm{~min}$ of exposure (Fig. 2). The expression levels of unphosphorylated ERK1/2 or unphosphorylated CREB remained unchanged, confirming equal loading of protein. The PACC cell line NCI-H322 demonstrated a similar response
$\mathbf{A}$

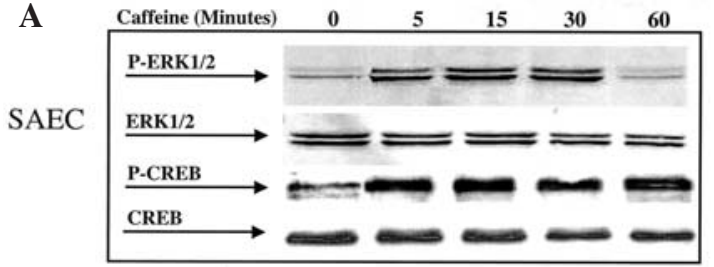

B

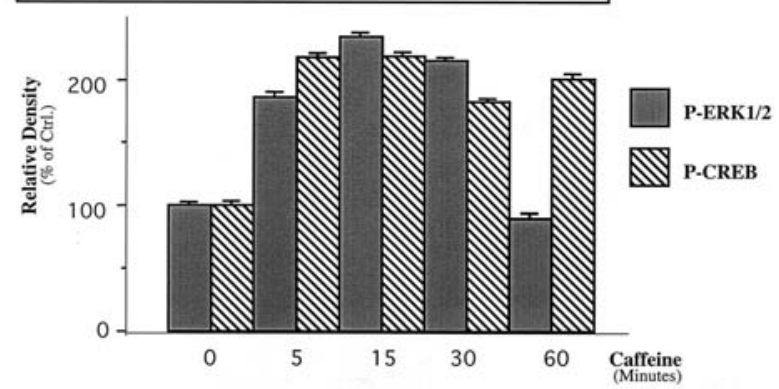

Figure 2. A, Western blot exemplifying the effects of caffeine (10 pM) on the expression of phosphorylated ERK1/2 and phosphorylated CREB in SAECs. The bands for P-ERK1/2 and P-CREB increased in size and density over time while the bands for total proteins (ERK1/2, CREB) remained unchanged. B, Bar graph illustrating normalized (controls are set as 100\%) mean values and standard deviations of five densitometric readings per band. The experiment was repeated twice with similar results. Statistical analysis was by one-way ANOVA, Tukey-Kramer multiple comparison test and two-tailed unpaired t-test. The increase in P-ERK1/2 was significant $(\mathrm{P}<0.001)$ at the 5-, 15- and 30-min time intervals while the observed increase in P-CREB was significant $(\mathrm{P}<0.001)$ at all time intervals tested.

A

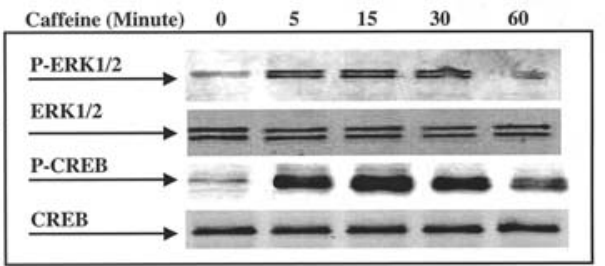

B

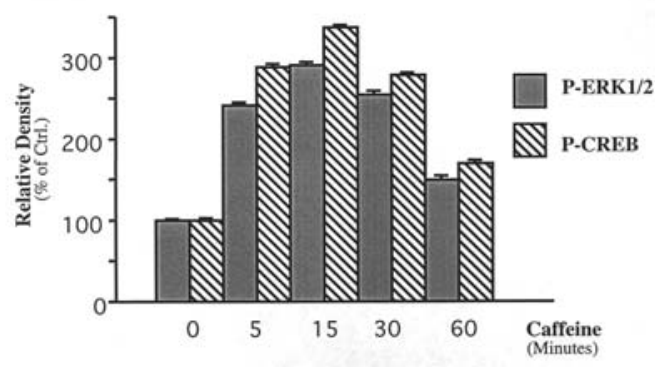

Figure 3. A, Western blot exemplifying the effects of caffeine $(10 \mathrm{pM})$ on the expression of phosphorylated ERK $1 / 2$ and phosphorylated CREB in NCI-H322. The bands for P-ERK1/2 and P-CREB increased in size and density over time while the bands for total proteins (ERK1/2, CREB) remained unchanged. B, Bar graph illustrating normalized (controls are set as $100 \%$ ) mean values and standard deviations of five densitometric readings per band. The experiment was repeated twice with similar results. Statistical analysis was by one-way ANOVA, Tukey-Kramer multiple comparison test and twotailed unpaired t-test. The increase in P-ERK1/2 and P-CREB was significant $(\mathrm{P}<0.001)$ at all time intervals tested.

to caffeine $(10 \mathrm{pM})$. Induction of P-ERK1/2 was highly significant $(\mathrm{P}<0.001)$ after 5,15 and $30 \mathrm{~min}$ of exposure to caffeine, with peak levels (2.8-fold increase) at the 15 -min time interval (Fig. 3) in these cells. Induction of phosphorylated CREB by caffeine in these PACC cells was even more 


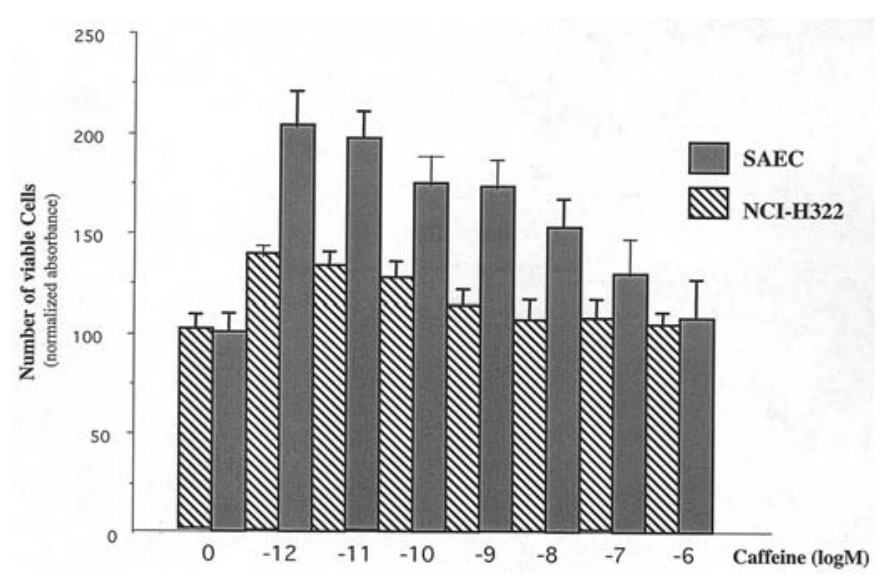

Figure 4. Effects of caffeine (1 pM-1 $\mu \mathrm{M})$ on cell number in SAECs as assessed by MTT assay. The cells were allowed to attach to the culture vessel surfaces in complete media at $37^{\circ} \mathrm{C}$ in $5 \% \mathrm{CO}_{2}$ for $5 \mathrm{~h}$. The cells were then switched to low serum medium and exposed for $72 \mathrm{~h}$ to caffeine at the concentrations indicated. Bars represent normalized (control values set at $100 \%$ ) mean values and standard errors of four samples per treatment group. The experiments were repeated twice with similar data. Statistical analysis of data was by one-way ANOVA, Tukey-Kramer multiple comparison test and two-tailed unpaired t-test. The observed increases in SAEC cell number were significant $(\mathrm{P}<0.001)$ at concentrations from $1 \mathrm{pM}$ to $10 \mathrm{nM}$. NCI-H322 cells were less responsive and yielded a small but significant $(\mathrm{P}<0.01)$ increase at the 1,10 and $100 \mathrm{pM}$ concentrations of caffeine.

pronounced ( $\mathrm{P}<0.001$ after 5,15 and $30 \mathrm{~min})$, with a 3.3 -fold increase after $15 \mathrm{~min}$ of exposure to caffeine.

Assessment of cell proliferation by MTT assays showed that caffeine increased the numbers of viable cells in both cell lines in a concentration-dependent manner after $72 \mathrm{~h}$ of exposure, with the lowest concentrations (1 pM, $10 \mathrm{pM})$ yielding the most pronounced responses (Fig. 4). NCI-H322 cells which demonstrated high base level proliferation in serum-free medium were less responsive than the immortalized SAECs. In both cell lines, the proliferative response to caffeine was highly significant $(\mathrm{P}<0.001)$ at the 4 lowest concentrations of caffeine (1 pM, $10 \mathrm{pM}, 100 \mathrm{pM}, 1 \mathrm{nM})$.

\section{Discussion}

The phosphodiesterase inhibitor caffeine is contained in coffee, tea, caffeinated beverages and in numerous dietary supplements that promise weight loss while boosting energy. Both, tea and caffeine have been shown to prevent the development of lung cancer in mice and rats $(18,19)$. However, the use of these agents for cancer prevention has recently been challenged by reports that showed a significant and selective promotion of NNK-induced PACC in hamsters by green tea or the phosphodiesterase inhibitor theophylline contained in tea while the development of neuroendocrine carcinomas was inhibited (12). Moreover, a significantly elevated lung cancer risk has recently been reported in individuals who consumed 2-3 cups of regular coffee daily (20). Experiments with the PACC cell line, NCIH322 and their putative normal cells of origin, SAECs, showed that theophylline stimulated the proliferation of both cell lines via cAMP-initiated activation of protein kinase A (PKA), mitogen-activated protein kinases ERK1/2 and cAMP response element binding protein (CREB) (13). Our current studies with caffeine in these two cell lines yielded similar responses. Low concentrations of caffeine that can be realistically expected in individuals who consume moderate amounts of coffee or tea significantly stimulated the proliferation of PACC and SAECs in MTT assays. Moreover, caffeine caused a significant and time-dependent increase in PKA activity, P-ERK1/2 and P-CREB. Collectively, these data suggest that caffeine may promote the development of PACC in humans.

These findings are in accord with our recently published observations that theophylline (13), ß-carotene (21) and dexamethasone (22) all stimulated the proliferation of PACC and SAECs in vitro via activation of PKA, CREB and ERK1/2. Consequently, all of these agents have to be considered as potential tumor promoters for the development of PACC. This interpretation contrasts sharply with the widely held belief that tea $(18,19)$, caffeine (19), glucocorticoids (23) and $\beta$-carotene $(24,25)$ may be useful as cancer preventive agents. We have recently shown that cAMP/PKA selectively stimulate the growth of SAECs and PACC while inhibiting the growth of large airway epithelial cells $(9,10,21)$. On the other hand, cAMP/PKA has been shown to inhibit the proliferation of small cell lung cancer cells (26) and PAC of alveolar type II cell phenotype (10). Collectively, these observations clearly illustrate that the cAMP/PKA pathway stimulates proliferation in some cells and the cancers derived from them while inhibiting others. Attempts to arrive at successful cancer preventive strategies as well as efforts to treat cancer with agents that inhibit components of signal transduction pathways have to take the highly cell type-specific function of signal transduction pathways into account. Unlike diagnostic tools are developed that allow for the identification of hyperactive pathways in each individual patient prior to the assignment to such therapies such efforts will continue to fail and may instead even promote the development of certain cancers.

\section{References}

1. Burns DM: Tobacco-related diseases. Semin Oncol Nurs 19: 244-249, 2003.

2. Hoffmann D, Rivenson A and Hecht SS: The biological significance of tobacco-specific N-nitrosamines: smoking and adenocarcinoma of the lung. Crit Rev Toxicol 26: 199-211, 1996.

3. Schuller HM: Mechanisms of smoking-related lung and pancreatic adenocarcinoma development. Nat Rev Cancer 2: 455-463, 2002.

4. Osann KE: Lung cancer in women: the importance of smoking, family history of cancer, and medical history of respiratory disease. Cancer Res 51: 4893-4897, 1991.

5. Linnoila I: Pathology of non-small cell lung cancer. New diagnostic approaches. Hematol Oncol Clin North Am 4: 1027-1051, 1990.

6. Broers JL, Jensen SM, Travis WD, Pass H, Whitsett JA, Singh G, Katyal SL, Gazdar AF, Minna JD and Linnoila RI: Expression of surfactant associated protein-A and Clara cell 10 kilodalton mRNA in neoplastic and non-neoplastic human lung tissue as detected by in situ hybridization. Lab Invest 66: 337-346, 1992 .

7. Albertine KH, Steiner RM, Radack DM, Golding DM, Peterson D, Cohn HE and Farber JL: Analysis of cell type and radiographic presentation as predictors of the clinical course of patients with bronchioalveolar cell carcinoma. Chest 113: 997-1006, 1998.

8. Linnoila RI, Szabo E, De Mayo F, Witschi H, Sabourin C and Malkinson A: The role of CC10 in pulmonary carcinogenesis: from a marker to tumor suppression. Ann NY Acad Sci 923: 249-267, 2000. 
9. Schuller HM, Tithof PK, Williams M and Plummer H III: The tobacco-specific carcinogen 4-(methylnitrosamino)-1-(3-pyridyl)1-butanone is a beta-adrenergic agonist and stimulates DNA synthesis in lung adenocarcinoma via beta-adrenergic receptormediated release of arachidonic acid. Cancer Res 59: 4510-4515, 1999.

10. Adissu HA and Schuller HM: Antagonistic growth regulation of cell lines derived from human lung adenocarcinomas of Clara cell and aveolar type II cell lineage: implications for chemoprevention. Int J Oncol 24: 1467-1472, 2004.

11. Schuller HM, Porter B and Riechert A: Beta-adrenergic modulation of NNK-induced lung carcinogenesis in hamsters. J Cancer Res Clin Oncol 126: 624-630, 2000.

12. Schuller HM, Porter B, Riechert A, Walker K and Schmoyer R: Neuroendocrine lung carcinogenesis in hamsters is inhibited by green tea or theophylline while the development of adenocarcinomas is promoted: implications for chemoprevention in smokers. Lung Cancer 45: 11-18, 2004.

13. Hussein A, Al-Wadei HAN, Takahashi T and Schuller HM: Theophylline stimulates cAMP-mediated signaling associated with growth regulation in human cells from pulmonary adenocarcinoma and small airway epithelia. Int J Oncol 27: 155-160, 2005.

14. Rall TW: Drugs used in the treatment of asthma. In: The Pharmacological Basis of Therapeutics. Goddman GA, Rall WT, Nies AS and Taylor P (eds). Pergamon Press, New York, pp618-637, 1990.

15. Daniel PB, Walker WH and Habener JF: Cyclic AMP signaling and gene regulation. Annu Rev Nutr 18: 353-383, 1998.

16. Kim J, Eckhart AD, Eguchi S and Koch WJ: beta-adrenergic receptor mediated DNA synthesis in cardiac fibroblasts is dependent on transactivation of the epidermal growth factor receptor and subsequent activation of extracellular signal-regulated kinases. J Biol Chem 277: 32116-32123, 2002.

17. Maudsley S, Pierce KL, Zamah AM, Miller WE, Ahn S, Daaka Y, Lefkowitz RJ and Luttrell LM: The beta(2)-adrenergic receptor mediates extracellular signal-regulated kinase activation via assembly of a multi-receptor complex with the epidermal growth factor receptor. J Biol Chem 275: 9572-9580, 2000.
18. Chung FL: The prevention of lung cancer induced by a tobaccospecific carcinogen in rodents by green and black Tea. Proc Soc Exp Biol Med 220: 244-248, 1999.

19. Chung FL, Wang M, Rivenson A, Iatropoulos MJ, Reinhardt JC, Pittman B. Ho CT and Amin SG: Inhibition of lung carcinogenesis by black tea in Fischer rats treated with a tobacco-specific carcinogen: caffeine as an important constituent. Cancer Res 58: 4096-4101, 1998.

20. Baker JA, McCann SE, Reid ME, Nowell S, Beehler GP and Moysich KB: Associations between black tea and coffee consumption and risk of lung cancer among current and former smokers. Nutr Cancer 52: 15-21, 2005.

21. Al-Wadei HAN, Takahashi T and Schuller H: Growth stimulation of human pulmonary adenocarcinoma cells and small airway epithelial cells by beta-carotene via activation of cAMP, PKA, CREB and ERK $1 / 2$. Int J Cancer (In press).

22. Al-Wadei HAN, Takahashi T and Schuller HM: PKA-dependent growth stimulation by dexamethasone of cells derived from human pulmonary adenocarcinoma and small airway epithelium. Eur J Cancer (In press).

23. Yao R, Wang Y, Lemon WJ, Lubet RA and You M: Budesonide exerts its chemopreventive efficacy during mouse lung tumorigenesis by modulating gene expressions. Oncogene 23: 7746-7752, 2004.

24. Cohen V and Khuri FR: Chemoprevention of lung cancer. Curr Opin Pulm Med 10: 279-283, 2004.

25. Furukawa F, Nishikawa A, Kasahara K, Lee IS, Wakabayashi K, Takahashi $\mathbf{M}$ and Hirose $\mathrm{M}$ : Inhibition by beta-carotene of upper respiratory tumorigenesis in hamsters receiving diethylnitrosamine followed by cigarette smoke exposure. Jpn J Cancer Res 90: 154-161, 1999.

26. Shafer SH, Phelps SH and Williams CL: Reduced DNA synthesis and cell viability in small cell lung carcinoma by treatment with cyclic AMP phosphodiesterase inhibitors. Biochem Pharmacol 56: 1229-1236, 1998. 